TRANSACTIONS OF THE

AMERICAN MATHEMATICAL SOCIETY

Volume 187, Is sue 1,1974

\title{
ELEMENTARY DIVISOR RINGS AND FINITELY PRESENTED MODULES
}

\author{
BY
}

\author{
MAX D. LARSEN, WILLIAM J. LEWIS AND THOMAS S. SHORES(1)
}

ABSTRACT. Throughout, rings are commutative with unit and modules are unital. We prove that $R$ is an elementary divisor ring if and only if every finitely presented module over $R$ is a direct sum of cyclic modules, thus providing a converse to a theorem of Kaplansky and answering a question of Warfield. We show that every Bezout ring with a finite number of minimal prime ideals is Hermite. So, in particular, semilocal Bezout rings are Hermite answering affirmatively a question of Henriksen. We show that every semihereditary Bezout ring is Hermite. Semilocal adequate rings are characterized and a partial converse to a theorem of Henriksen is established.

1. Introduction. All rings considered will be commutative and have identity; all modules will be unital. A ring is a Bezout ring if every finitely generated ideal is principal. An $R$-module $M$ is finitely presented if $M \cong R^{(n)} / K$ for some finitely generated submodule $K$ of the free module $R^{(n)}$. Finitely presented modules can be interpreted matricially (see remarks at the beginning of $\$ 3$ ). Thus a study of decompositions of finitely presented modules leads one to the study of matrices over rings.

Following Kaplansky [11] a ring is said to be an elementary divisor ring if every matrix over $R$ is equivalent to a diagonal matrix. As a part of a study of elementary divisor rings and other related types of rings, Kaplansky proved that if $R$ is an elementary divisor ring then every finitely presented $R$-module is a direct sum of cyclic modules.

In $\$ 3$ we prove the converse of this theorem, thus answering a question of Warfield [19]. A ring is said to be Hermite if every 1 by 2 and 2 by 1 matrix over it is equivalent to a diagonal matrix. We show that every Bezout ring with a finite number of minimal prime ideals is Hermite. In particular this shows that a semilocal Bezout ring is Hermite and provides an affirmative answer to a question of Henriksen [7].

Presented to the Society, J anu ary 26, 1973 under the titles Elementary divisor rings and Bezout rings which are Hermite; received by the editors November 10, 1972.

AMS (MOS) subject classifications (1970). Primary 13C05; Secondary 13 F05.

Key words and phrases. Bezout rings, finitely presented modules, Hermite rings, elementary divisor rings, adequate rings.

(1) The first two named authors received financial support during the writing of this paper from the University of Nebraska Research Council; the former held a Maude Hammond Fling Summer Fellowship and the latter a Junior Faculty Summer Fellowship. 
In addition we show that semihereditary Bezout rings are Hermite.

A ring $R$ is said to be adequate if $R$ is Bezout and for $a, b \in R$ with $a \neq 0$, there exist $r, s \in R$ such that $a=r s,(r, b)=(1)$, and if a nonunit $s^{\prime}$ divides $s$, then $\left(s^{\prime}, b\right) \neq(1)$. Adequate rings have been studied by Helmer [6], Kaplansky [11] and Gillman and Henriksen [3], [4], [7]. We obtain a complete characterization of semilocal adequate rings and a partial converse to a theorem of Henriksen [7] concerning the prime ideal structure of an adequate ring.

The Jacobson radical of a ring $R$ will be denoted by $J(R)$. The terms semilocal and local will not imply chain conditions. The ring itself will not be considered a prime ideal. Following Kaplansky the term valuation ring will refer to a ring, not necessarily an integral domain, in which given any two elements, one divides the other. The use of the word domain will imply that there are no zero divisors (e.g., a Bezout domain is a Bezout ring with no zero divisors).

2. Hermite rings. We shall call two matrices $A$ and $B$ over a ring $R$ equivalent (notation: $A \sim B$ ) if there exists invertible matrices $P, Q$ such that $B=P A Q$. An $m$ by $n$ matrix $A$ admits diagonal reduction if $A$ is equivalent to a diagonal matrix $\left[d_{i j}\right.$ ] (i.e. $d_{i j}=0$ whenever $i \neq j$ ) with the property that every $d_{i i}$ is a divisor of $d_{i+1, i+1}$. If every 1 by 2 and 2 by 1 matrix over $R$ admits diagonal reduction then $R$ is an Hermite ring. If every matrix over $R$ admits diagonal reduction then $R$ is an elementary divisor ring.

Hermite rings and elementary divisor rings were introduced by Kaplansky in [11]. Obviously an elementary divisor ring is Hermite and it is easy to see that an Hermite ring is Bezout. Examples that neither implication is reversible are provided by Gillman and Henriksen in [3]. In the papers mentioned above as well as in [4] and [7], effort has been made to find conditions under which the notions of Bezout ring and Hermite ring are equivalent. Also pursued is a search for conditions under which an Hermite ring will be an elementary divisor ring. This section is concerned with the same problems.

In [11, Theorem 3.2, p. 467] Kaplansky proved that a Bezout ring is Hermite when all the zero divisors of the ring are in the Jacobson radical, establishing in particular the fact that all Bezout domains are Hermite. Henriksen [7, Theorem 2, p. 160] changed Kaplansky's hypothes is to the assumption that the Jacobson radical contains a prime ideal and proved that the theorem is still valid.

A Bezout ring with either of these two conditions on its Jacobson radical becomes quite special. In fact, all that is needed is that a ring be arithmetical [9], (i.e. the localization at each maximal ideal is a valuation ring). A Bezout ring is, of course, arithmetical. Let us consider the following conditions for an arithmetical ring $R$ :

(i) All zero divisors of $R$ are contained in $J(R)$.

(ii) The set of zero divisors of $R$ is a prime ideal contained in $J(R)$. 
(iii) $J(R)$ contains a prime ideal.

(iv) $R$ has a unique minimal prime ideal.

In an arithmetical ring incomparable primes are comaximal [9, Corollary 2, p. 116], and in any ring the zero divisors are a union of prime ideals. Using these two facts one can see that (i) and (ii) are equivalent, and they imply (iii) which is equivalent to (iv). An example of a Bezout ring which satisfies (iii) but not (i) can be found as follows. Let $R$ be a Bezout domain with exactly two maximal ideals $M_{1}$ and $M_{2}$ and one nonzero prime $P \subset M_{1} \cap M_{2}$. If $m \in M_{1} \backslash M_{2}, 0 \neq p \in P$, and $q=p m$, then the example is provided by the ring $R / q R$. Another example is provided by taking the direct product of $p^{\infty}$ - and $q^{\infty}$-groups of Prüfer type where $p, q$ are distinct primes, endowing it with the trivial multiplication, and adjoining an identity.

A slight generalization of the proof of $[7$, Theorem 2, p. 160] will prove the following. Here $(0: d)=\{x \in R \mid x d=0\}$.

(2.1) Proposition. A Bezout ring $R$ is Hermite if $(0: d) \subseteq J(R)$ for all $d \notin J(R)$.

We do not know whether or not this condition is actually weaker for arithmetical rings than Henriksen's hypothesis discussed earlier (i.e., $J(R)$ contains a prime ideal).

We now prove the equivalence of Bezout and Hermite under less stringent hypotheses than (i)-(iv) considered above.

(2.2) Theorem. Every Bezout ring $R$ with a finite number of minimal prime ideals is Hermite.

Proof. Let $P(R)$ be the prime radical of $R$, and let $P_{1}, \ldots, P_{n}$ be the minimal prime ideals of $R$. Since every Bezout ring is arithmetical, the $P_{i}$ 's are pairwise comaximal, and hence $R / P(R)$ is a direct sum of integral domains $R / P_{1}, \cdots, R / P_{n}$. Thus there exist mutually orthogonal idempotents $\bar{e}_{1}, \ldots, \bar{e}_{n}$ with $\bar{e}_{i} \in R / P_{i}$ such that $\bar{e}_{1}+\cdots+\bar{e}_{n}=\overline{1}$. Lift them to mutually orthogonal idempotents $e_{1}, \cdots$, $e_{n} \in R$ [14, Propositions 1 and 2, p. 73]. Since $1-\left(e_{1}+\cdots+e_{n}\right)$ is an idempotent element in $P(R)$, it must be zero. Thus $R=e_{1} R \oplus \cdots \oplus e_{n} R$ and each $e_{i} R$ has a unique minimal prime ideal. Each $e_{i} R$ is a homomorphic image of $R$, so is Bezout. Thus Henriksen's theorem says that each $e_{i} R$ is Hermite. Now use coordinatewise manipulations to show that $R$ satisfies condition $(T)$ of Gillman and Henriksen [4, Theorem 3, p. 363] and conclude that $R$ is Hermite.

As a corollary to Theorem 2.2 we obtain an affirmative answer to a question of Henriksen posed in [7, Question 3, p. 162]. This solution (actually (2) implies (3) in the corollary below) provides the final link in the equivalence below. 
(2.3) Corollary. The following are equivalent for a semilocal ring $R$ :

(1) $R$ is an aritbmetical ring.

(2) $R$ is a Bezout ring.

(3) $R$ is an Hermite ring.

(4) $R$ is an elementary divisor ring.

(5) Every finitely presented module over $R$ is a direct sum of cyclic modules.

Proof. The implications (4) $\Rightarrow(3) \Rightarrow(2) \Rightarrow(1)$ and $(4) \Rightarrow(5)$ are true without the semilocal hypothesis [11] as is $(5) \Rightarrow(1)$ [19, Theorem 3, p. 169]. Let $R$ be a semilocal arithmetical ring. By $[9$, Theorem 5, p. 119] $R$ is a Bezout ring. Since $R$ is arithmetical, each maximal ideal contains a unique minimal prime ideal; hence there are finitely many minimal prime ideals. The theorem now says that $R$ is Hermite and [7, Corollary 2, p. 161] can be applied to conclude that $R$ is an elementary divisor ring.

We provide one final condition under which a Bezout ring is Hermite. Recall that a ring is semibereditary if every finitely generated ideal is projective.

(2.4) Theorem. Every semibereditary Bezout ring is an Hermite ring.

Proof. Let $R$ be a semihereditary Bezout ring and let $a, b \in R$ with $(a, b)=$ (d). We will verify that condition ( $T$ ) of Gillman and Henriksen [4, Theorem 3, p. 363] holds. Set $K=(0: d)$ and obtain from the projectivity of $(d)$ that the exact sequence $0 \rightarrow K \rightarrow R \rightarrow(d) \rightarrow 0$ splits, whence $R=K \oplus I$ for some ideal $I$. Write $d=k+i$ for some $k \in K, i \in I$ and from the equation $k d=0$ and the directness of the sum obtain the fact that $k^{2}=0$. Since semihereditary rings have no nontrivial nilpotent elements $k=0$ and $d \in I$. Thus $a, b \in I$. Write $1=e+f$ for some $e \in K, f \in I$, and also write $a=a^{\prime} d, b=b^{\prime} d$ where $a^{\prime}, b^{\prime} \in I$. For some $m, n \in R$ we have $m a+n b=d$, so by separation into components of $K$ and $I$ we obtain $m^{\prime} a+n^{\prime} b=d$ for suitable $m^{\prime}, n^{\prime} \in I$. Thus $m^{\prime} a^{\prime} d+n^{\prime} b^{\prime} d=d$ and hence $\left(f-m^{\prime} a^{\prime}-n^{\prime} b^{\prime}\right) d=0$. Thus $f-m^{\prime} a^{\prime}-n^{\prime} b^{\prime} \in K \cap I=0$, so $\left(a^{\prime}, b^{\prime}\right)=I$. Now set $a_{1}=a^{\prime}$ and $b_{1}=e+b^{\prime}$; then $a=a_{1} d, b=b_{1} d$, and $\left(a_{1}, b_{1}\right)=\left(a^{\prime}, b^{\prime}, e\right)=(1)$.

It is an open question [7, Question 1, p. 162] whether or not every Bezout domain is an elementary divisor ring. More generally, we might ask whether or not every semihereditary Bezout ring is an elementary divisor ring. We now turn to a consideration of conditions under which an Hermite ring will be an elementary divisor ring.

Gillman and Henriksen [4, Theorem 6, p. 364] prove that $R$ is an elementary divisor ring if and only if $R$ is an Hemite ring and satisfies the following condition:

(D') For all $a, b, c \in R$ with $(a, b, c)=(1)$, there exist $p, q \in R$ such that $(p a, p b+q c)=(1)$. 
Their proof is an adaptation of Kaplansky's proof [11, Theorem 5.2, p. 472] that if the zero divisors of a ring $R$ are in $J(R)$, then $R$ is an elementary divisor ring if and only if $R$ is Bezout and satisfies ( $\left.D^{\prime}\right)$.

The following condition leads to a common generalization of $[4$, Theorem 8 , p. 364] and [7, Theorem 5, p. 161].

For $a, b \in R$ with $a \notin J(R)$, there exists $m \in R$ such that $(b, m)=(1)$

and if for some $n \in R(n, a) \neq(1)$ and $(n, b)=(1)$, then $(n, m) \neq(1)$.

(2.5) Theorem. If $R$ is a Bezout ring satisfying $(\mathrm{N})$, then $R$ satisfies $\left(D^{\prime}\right)$.

Proof. Let $a, b, c \in R$ with $(a, b, c)=(1)$. If $a \in J(R)$, then $(b, c)=(1)$ and we may choose $p, q$ such that $p b+q c=1$. Thus we assume $a \notin J(R)$ and pick $m \in R$ such that $(b, m)=(1)$, and if for some $n \in R(n, a) \neq(1)$ and $(n, b)=(1)$ then $(n, m) \neq(1)$.

We note that if $d$ is a nonunit and $d \mid a$ then either $(d, b) \neq(1)$ or $(d, m) \neq(1)$. Now if we assume that $d$ is a nonunit and $(d)=(a, b+m c)$ then there is a nonunit $d^{\prime} \mid d$ such that $d^{\prime}\left|a, d^{\prime}\right|(b+m c)$, and either $d^{\prime} \mid b$ or $d^{\prime} \mid m c$ hence both. We shall show that this is impossible. Indeed if $d^{\prime}\left|a, d^{\prime}\right| b$ then $(b, m)=(1)$ implies $\left(d^{\prime}, m\right)=(1)$ and $(a, b, c)=(1)$ implies $\left(d^{\prime}, c\right)=(1)$. Hence $\left(d^{\prime}, m c\right)=(1)$ and we have the desired contradiction.

Using the result of Gillman and Henriksen [4, Theorem 6, p. 364] we immediately get the following

(2.6) Corollary. Every Hermite ring satisfying $(\mathrm{N})$ is an elementary divisor ring.

The most striking class of rings which satisfies $(\mathrm{N})$ is the class of adequate rings. More discussion of adequate rings occurs in $\$ 4$; now we show only that $(\mathrm{N})$ holds in an adequate ring. Let $a, b \in R$, an adequate ring, with $a \neq 0$. Write $a=r s$ according to the adequate condition. Then $r$ plays the part of $m$ in condition $(\mathrm{N})$. Certainly $(r, b)=(1)$. If $(n, a) \neq(1)$ and $(n, b)=(1)$ for some $n$, one can check that $(n, s)=(1)$ and thus $(n, r) \neq(1)$.

Let $Z(a)$ denote the set of maximal ideals containing $a$. Henriksen [7, Theorem 5, p. 161] proves that an Hermite ring $R$ is an elementary divisor ring if for every $a, b \in R$ with $a \notin J(R)$ there exists $r \in R$ such that $Z(r)=Z(a) \backslash Z(b)$. Rings satisfying this hypothesis are another example of rings that satisfy $(\mathrm{N})$. Indeed, if $Z(r)=Z(a) \backslash Z(b)$ we surely have $(r, b)=(1)$. Also if $(n, a) \neq(1)$ and $(n, b)=(1)$ for some $n$ then $\varnothing \neq Z(a) \cap Z(n) \subseteq Z(r)$; so $(n, r) \neq(1)$.

We note that in our proof of Theorem 2.3 we established a condition slightly stronger than $\left(D^{\prime}\right)$, that is, if $a \notin J(R)$ and $(a, b, c)=(1)$, there exists $m \in R$ such that $(a, b+m c)=(1)$. This condition, for a Bezout ring, defines a $B$-ring as studied in [16]. Thus a Bezout ring satisfying $(\mathrm{N})$ is a $B$-ring. 
Finally our Corollary 2.3 allows the more desirable wording of Henriksen's Corollary 2 [7, p. 161].

(2.7) Proposition. If $R$ is a Bezout ring and $Z(a)$ is finite for each $a \notin J(R)$, then $R$ is an elementary divisor ring.

3. Elementary divisor rings. In this section a number of results about Bezout rings are established. These results provide major links between Bezout rings, Hermite rings, elementary divisor rings, and rings whose finitely presented modules are direct sums of cyclic modules.

We begin with some remarks on naming of finitely presented modules by matrices. If $\phi \in \operatorname{Hom}_{R}\left(R^{(n)}, R^{(m)}\right)$ we say that $\phi$ names the module $M$ if the sequence

$$
R^{(n)} \stackrel{\phi}{\longrightarrow} R^{(m)} \longrightarrow M \longrightarrow 0
$$

is exact, i.e., $M \cong \operatorname{coker}(\phi)$. The matrix representation of $\phi$ with respect to bases $\left\{e_{1}, \cdots, e_{n}\right\}$ and $\left\{f_{1}, \cdots, f_{m}\right\}$ of $R^{(n)}$ and $R^{(m)}$, respectively, is the matrix $A=\left[a_{i j}\right]$ where $\phi\left(e_{i}\right)=a_{1 i} f_{1}+\cdots+a_{m i} f_{m}$ for $i=1, \cdots, n$. Now let $A$ be an $m$ by $n$ matrix over $R$. Say $A$ names $M$ (with respect to bases $E, F$ of $R^{(n)}$, $R^{(m)}$, respectively) if $A$ is the matrix representation of a homomorphism $\phi$ with respect to the bases $E, F$ and $\phi$ names $M$. Initially we can afford to be careless about bases; for, if $A$ names $M$ with respect to bases $E, F$ and names $M^{\prime}$ with respect to bases $E^{\prime}, F^{\prime}$, then $M$ is isomorphic to $M^{\prime}$. Thus when we say $A$ names $M$ without reference to specific bases it will be understood that we mean the canonical bases. Let $E, F$ be bases of $R^{(n)}, R^{(m)}$, respectively, and let $Q^{-1}$, $P$ be change of bases matrices which convert $E$ to $E^{\prime}$ and $F$ to $F^{\prime}$ respectively. If $A$ names $M$ with respect to $E, F$, then $P A Q$ names $M$ with respect to $E^{\prime}, F^{\prime}$.

(3.1) Theorem. All diagonal matrices over a ring $R$ admit diagonal reduction if and only if $R$ is a Bezout ring.

Proof. If $a, b \in R$ and the matrix

$$
\left[\begin{array}{ll}
a & 0 \\
0 & b
\end{array}\right]
$$

admits diagonal reduction, say

$$
\left[\begin{array}{ll}
a & 0 \\
0 & b
\end{array}\right] \sim\left[\begin{array}{ll}
d & 0 \\
0 & e
\end{array}\right]
$$

where $d$ divides $e$, then $(a, b)=(d, e)=(d)$, so that $R$ is a Bezout ring.

To establish the converse let $R$ be a Bezout ring and induct on $m$ to show that the diagonal $m$ by $n$ matrix $A$ admits diagonal reduction. The case $m=1$ offers no difficulty. If $m>1$, write

$$
A=\left[\begin{array}{cc}
a & 0 \\
0 & A_{1}
\end{array}\right]
$$


where $A_{1}$ is an $m-1$ by $n-1$ diagonal matrix. By the induction hypothesis, $A_{1}$ admits diagonal reduction:

$$
A_{1} \sim\left[\begin{array}{lllll}
c_{1} & 0 & \cdots & \cdot & 0 \\
0 & c_{2} & \cdot & \cdot & 0 \\
\cdot & \bullet & \cdot & & \\
\cdot & \cdot & & \ddots &
\end{array}\right] .
$$

Note that if $d=\left(a, c_{1}\right)$, say $d=m a+n c_{1}, a=d a^{\prime}$ and $c_{1}=d c_{1}^{\prime}$, then use of elementary row and column operations yield the equivalences

$$
\left[\begin{array}{ll}
a & 0 \\
0 & c_{1}
\end{array}\right] \sim\left[\begin{array}{rr}
a & m a+n c_{1} \\
0 & c_{1}
\end{array}\right] \sim\left[\begin{array}{ll}
d & a \\
c_{1} & 0
\end{array}\right] \sim\left[\begin{array}{rr}
d & 0 \\
0 & -a^{\prime} c_{1}
\end{array}\right] .
$$

Hence

$$
A \sim\left[\begin{array}{cccccc}
d & 0 & 0 & \cdots & 0 \\
0 & -a^{\prime} c_{1} & 0 & \cdots & 0 & 0 \\
0 & 0 & c_{2} & \cdots & 0 \\
\cdot & \cdot & & \cdot & & \\
\cdot & \cdot & & & &
\end{array}\right]
$$

Since $d$ divides $c_{1}, d$ must divide all the diagonal entries. Apply induction again to the matrix obtained by deleting the first row and column of the right-hand side to obtain the proper form.

There is some interest in knowing when the intersection of principal ideals is itself principal; for instance, see Lemma 3.3 and Theorem 3.4 of Kaplansky [11, p. 468]. The following corollary shows that such is always the case in a (commutative) Bezout ring.

(3.2) Corollary. The intersection of two principal ideals in a Bezout ring is itself principal.

Proof. Let $a, b \in R$. Then

$$
\left[\begin{array}{ll}
a & 0 \\
0 & b
\end{array}\right] \sim\left[\begin{array}{ll}
d & 0 \\
0 & e
\end{array}\right]
$$

where $d$ divides $e$. If $M$ is the module named by either of these equivalent matrices, then we see that $e R=0: M=\{f \in R \mid a$ and $b$ divide $f\}=a R \cap b R$.

A module $M$ over $R$ will be said to bave a canonical form if $M \cong R / A_{1} \oplus \ldots$ $\oplus R / A_{n}$ where $A_{1}, \cdots, A_{n}$ are ideals of $R$ and $A_{1} \subseteq A_{2} \subseteq \cdots \subseteq A_{n} \neq R$. Kaplansky shows in Theorems 9.2 and 9.3 of $[11$, p. 478] that if $M$ has another canonical form $M=R / B_{1} \oplus \cdots \oplus R / B_{m}$, then $n=m$ and each $A_{i}=B_{i}$, and if $M$ has a canonical 
form with $n$ summands as above, then every generating set of $M$ requires at least $n$ elements.

Recall that a cyclic $R$-module of the form $R / r R$, where $r \in R$, is called a cyclically presented $R$-module. Using Bourbaki $[1, \mathrm{p} .37]$ one can show that a finitely presented cyclic module over a Bezout ring is cyclically presented.

(3.3) Corollary. If the ring $R$ is a Bezout ring, then every finitely presented module over $R$ which is a direct sum of cyclic modules bas a canonical form.

Proof. Since finitely presented cyclic modules over $R$ are cyclically presented, a finitely presented module over $R$ which is a direct sum of cyclic modules is named by a (square) diagonal matrix $A$. Also $A$ admits diagonal reduction by Theorem 3.1, say

$$
A \sim\left[\begin{array}{llllll}
d_{1} & 0 & \cdot & \cdot & 0 \\
0 & d_{2} & \cdot & \cdot & 0 & 0 \\
\cdot & \cdot & \cdot & & \\
\cdot & \cdot & & \cdot & & \\
\cdot & \cdot & & & \cdot &
\end{array}\right]
$$

where each $d_{i}$ divides $d_{i+1}$. Since equivalent matrices name isomorphic modules it follows that the module named by $A$ is isomorphic to $R / d_{1} R \oplus \cdots \oplus R / d_{n} R$, and reversing the order of the summands produces the canonical form.

Two lemmas precede our next theorem, Theorem 3.6. The second lemma as well as the theorem were announced without proof by Lafon [13, Théorème 1 and Proposition 2, p. 964]. We have been unable to obtain copies of his proofs and for the sake of completeness have included our own proofs.

(3.4) Lemma. Let $M$ be a finitely presented $R$-module and let $K$ be an ideal of $R$ contained in $0: M$ Then $M$ is a finitely presented $(R / K)$-module.

Proof. Let $M$ be named by the $m$ by $n$ matrix $A$. Then the $n$ columns of $A$ span the kernel of the map $\phi$ where the exact sequence

$$
R^{(n)} \stackrel{A}{\longrightarrow} R^{(m)} \stackrel{\phi}{\longrightarrow} M \longrightarrow 0
$$

gives $M$. Tensoring this sequence with $R / K$, we get an exact sequence

$$
(R / K)^{(n)} \rightarrow(R / K)^{(m)} \rightarrow M / K M \rightarrow 0 .
$$

Since $K \subseteq(0: M), M / K M=M$, and $M$ is a finitely presented $R / K$ module.

(3.5) Lemma. Let $R$ be an arithmetical ring and let $M$ be a faithful finitely presented $R$-module which is a direct sum of cyclic modules. Then $M$ bas a nonzero free summand. 
Proof. Write $M=R / A_{1} \oplus \cdots \oplus R / A_{n}$ for ideals $A_{i}$ of $R$ and argue by induction on $n$. The case $n=1$ is clear. Let $n>1$ and write $M^{\prime}=R / A_{1} \oplus \ldots \oplus R / A_{n-1}$. If $K=0: M^{\prime}$ then by Lemma $3.4 M^{\prime}$ is a faithful finitely presented $(R / K)$-module. If $K=0$ the proof is complete. If not induction at least gives $M^{\prime}=P \oplus R / K$ for some $P$, whence $M=P \oplus\left(R / K \oplus R / A_{n}\right)$. Therefore $(0: M)=K \cap A_{n}=0$. Since $R / K$ is finitely presented (since $M$ is) we have that $K$ is finitely generated as is $A_{n}$. Thus by arithmeticity and Theorem 3 of Jensen $[9$, p. 117]

$$
(0: K)+\left(0: A_{n}\right)=\left(0:\left(K \cap A_{n}\right)\right)=R \text {, }
$$

say $1=a+b$ for some $a \in(0: K), b \in\left(0: A_{n}\right)$. Now map $R / K \oplus R / A_{n}$ onto $R$ by $\left(r+K, s+A_{n}\right) \mapsto r a+s b$ to get the desired result.

(3.6) Theorem. Suppose that every module over $R$ which is named by a matrix of the form

$$
\left[\begin{array}{ll}
a & 0 \\
b & 0
\end{array}\right]
$$

is a direct sum of cyclic modules. Then $R$ is Hermite.

Proof. First we show that $R$ is arithmetical. Let $P$ be a maximal ideal of $R$ and let $\phi$ be the map with matrix representation

$$
\left[\begin{array}{ll}
a & 0 \\
b & 0
\end{array}\right]
$$

Tensoring the exact sequence

$$
R^{(2)} \stackrel{\phi}{\longrightarrow} R^{(2)} \stackrel{\psi}{\longrightarrow} M \longrightarrow 0
$$

with $R_{P}$ shows that the cokernel of the map induced by $\phi$ is $R_{P} \otimes M$ and that it must be a direct sum of cyclic $R_{P}$-modules. Since $R_{P}$ satisfies the hypotheses of the theorem, for simplicity of notation we temporarily assume $R=R_{P}$. Assume $R$ is not a valuation ring; then there are elements $a, b \in R$ such that neither divides the other. Let $A=(a R \cap b R)+a P+b P$; then $A \varsubsetneqq(a, b) \subseteq P$. Consider the exact sequence

$$
(R / A)^{(2)} \stackrel{\left[\begin{array}{cc}
a+A & 0 \\
b+A & 0
\end{array}\right]}{\longrightarrow}(R / A)^{(2)} \longrightarrow \bar{M} \longrightarrow 0 .
$$

In the proof of Theorem 2 of $[19$, p. 168], Warfield shows that $\bar{M}$ is indecomposable. However, this exact sequence is obtained by tensoring the first sequence with $R / A$. So using the canonical isomorphism $(R / A) \otimes M \cong M / A M$ one sees that $\bar{M} \cong M / A M$. Hence $M$ is indecomposable; for if $M=N \oplus K$ for nonzero submodules $N$ and $K$, then $M / A M \cong N / A N \oplus K / A K$. But by Nakayama's lemma, neither summand is trivial. This contradiction and the hypotheses say that $M$ is cyclic. Pulling a generator back to $R^{(2)}$ yields a generating set for $R^{(2)}$ of the form 


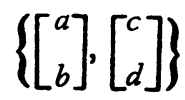

for some $c, d \in R$. But $\left[{ }_{b}^{a}\right] \in P R^{(2)}$ so $R^{(2)} / P R^{(2)}$ is cyclic. The isomorphism $R^{(2)} / P R^{(2)} \simeq(R / P)^{(2)}$ provides the necessary contradiction and we conclude that the local ring $R$ is a valuation ring and the original ring is arithmetical.

Now we return to the original notation and show that if $M$ is named by

$$
\left[\begin{array}{ll}
a & 0 \\
b & 0
\end{array}\right]
$$

then $M$ is faithful. An element $d$ annihilates $M$ if and only if

$$
\left[\begin{array}{l}
d \\
0
\end{array}\right],\left[\begin{array}{l}
0 \\
d
\end{array}\right] \in R\left[\begin{array}{l}
a \\
b
\end{array}\right]
$$

say $a a=d, \alpha b=0, \beta a=0$, and $\beta b=d$ for $\alpha, \beta \in R$. Pass to localizations. If $a$ divides $b$, say $b=a b_{1}$, then the above equations give $0=\beta a=\beta a b_{1}=\beta b=d$ locally. Likewise if $b$ divides $a$ in the localization, then $d=0$ there. Hence $d=0$ and $M$ is faithful.

The proof can now be completed. Let $M, a, b$ be as above. By Lemma 3.5 we write $M=e R \oplus Q$ where $R$ is free on $e$. We have the sequence

$$
R^{(2)} \stackrel{\psi}{\longrightarrow} e R \oplus Q \stackrel{\pi}{\longrightarrow} e R
$$

where $\pi$ is the projection and $\psi$ is the map onto $M$ in the sequence naming $M$. If $\sigma$ is their composition, then we have that the sequence

$$
R^{(2)} \stackrel{\sigma}{\longrightarrow} e R \longrightarrow 0
$$

splits, so $R^{(2)} \cong e R \oplus \operatorname{ker} \sigma$. By the proof of [15, Theorem 48, p. 143] ker $\sigma \simeq R$. Pick a basis $\left\{\epsilon_{1}, \epsilon_{2}\right\}$ of $R^{(2)}$ such that ker $\sigma=\epsilon_{1} R$ and $\psi\left(\epsilon_{2}\right)=e$. One has that $\operatorname{Im} \phi=\operatorname{ker} \psi \subseteq \operatorname{ker} \sigma=\epsilon_{1} R$. Consequently with respect to bases $B_{1}$ and $B_{2}$ where $B_{1}$ is the canonical basis and $B_{2}=\left\{\epsilon_{1}, \epsilon_{2}\right\}$ the matrix representation of $\phi$ has the following form:

$$
\left[\begin{array}{ll}
x & y \\
0 & 0
\end{array}\right]
$$

But this matrix is just $P A$ where $P$ is the change of basis matrix. A simple computation shows that for some $d \in R$

$$
P A=\left[\begin{array}{ll}
d & 0 \\
0 & 0
\end{array}\right]
$$

whence

$$
[a, b] P^{T}=[d, 0]
$$

so $R$ is Hermite.

This theorem has an interesting corollary in the spirit of [11, Theorem 5.1, p. 471]. 
(3.7) Corollary. The ring $R$ is an elementary divisor ring if (and only if) every 2 by 2 matrix over $R$ is equivalent to a diagonal matrix.

Proof. We need first to look at 1 by 2 and 2 by 1 matrices over $R$. Since $R$ is commutative we need only be concerned with the 2 by 1 matrices. Let $M$ be named by

$$
\left[\begin{array}{ll}
a & 0 \\
b & 0
\end{array}\right]
$$

Since this matrix is equivalent (by hypothesis) to a diagonal matrix, $M$ is a direct sum of cyclic modules. The theorem now says that $R$ is Hermite. Thus each 2 by 1 matrix over $R$ is equivalent to a diagonal matrix. Furthermore, $R$ is Bezout and thus all diagonal matrices admit diagonal reduction. Thus all 1 by 2,2 by 1 , and 2 by 2 matrices over $R$ admit diagonal reduction and the proof is complete by Kaplansky's theorem [11, Theorem 5.1, p. 471].

We are now able to prove the converse to a theorem proved by Kaplansky in 1949 [11, Theorem 9.1, p. 477] thus completely characterizing those rings whose finitely presented modules are a direct sum of cyclic modules. In 1970 Warfield showed in [19, Theorem 1 and Corollary 2.1, p. 168] that valuation rings are precisely the local rings whose finitely presented modules are direct sums of cyclic modules and concluded by stating (p. 171) that the global problem was still unsolved, even for domains. We are now able to provide a solution. Lafon has informed us that he has obtained this equivalence for rings whose zero divisors are contained in the Jacobson radical of the ring.

(3.8) Theorem. The ring $R$ is an elementary divisor ring if (and only if) every finitely presented $R$-module is a direct sum of cyclic modules.

Proof. By Theorem 3.6 and [4, Theorem 6, p. 364] it is sufficient to show that condition $\mathrm{D}^{\prime}$ (of the same theorem) is satisfied. To that end, let $a, b, c$ be elements of $R$ such that $(a, b, c)=(1)$. Let $M$ be the module named by the matrix

$$
A=\left[\begin{array}{ll}
a & 0 \\
b & c
\end{array}\right]
$$

and obtain from the hypotheses and Corollary 3.3 the fact that $M$ has a canonical form $R / d R \oplus R / e R$ where $d$ divides $e$. The second Fitting invariant of $M$ is just the ideal generated by the entries of any 2 by 2 matrix which names $M$. This ideal depends only on $M$ (see, for example, [10, Appendix 4-3(b), p. 145]) and both

$$
\left[\begin{array}{ll}
a & 0 \\
b & c
\end{array}\right],\left[\begin{array}{ll}
d & 0 \\
0 & e
\end{array}\right]
$$

name $M$, so $(1)=(a, b, c)=(d, e)=(d)$. It follows that $M \simeq R / e R$, i.e., $M$ is cyclic. If $K$ is the column space of $A$, select an element

$$
\left[\begin{array}{l}
u \\
v
\end{array}\right]
$$


in $R^{(2)}$ such that its image in $R^{(2)} / K$ generates $M$. Since $R$ is Hermite, by [4, Theorem 3, p. 363] one may find $d, x, y \in R$ such that

$$
d\left[\begin{array}{l}
x \\
y
\end{array}\right]=\left[\begin{array}{l}
u \\
v
\end{array}\right]
$$

and $(x, y)=(1)$. The image of

$$
\left[\begin{array}{l}
x \\
y
\end{array}\right]
$$

generates $M$ so that $R^{(2)}$ can be generated by

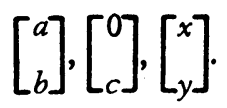

In particular note that for some $m, n \in R, m x+n y=1$. Also for suitable $p, q, r \in R$ we obtain that

$$
\left[\begin{array}{c}
-n \\
m
\end{array}\right]=p\left[\begin{array}{l}
a \\
b
\end{array}\right]+q\left[\begin{array}{l}
0 \\
c
\end{array}\right]+r\left[\begin{array}{l}
x \\
y
\end{array}\right] .
$$

Consequently it follows that $1=(p b+q c+r y) x-(p a+r x) y=(p b+q c) x-(p a) y$. Hence $(p a, p b+q c)=(1)$ and condition $D^{\prime}$ is satisfied.

The study of rings whose finitely presented modules are direct sums of cyclic modules has been interpreted matricially in terms of Fitting equivalence. Two matrices $A, B$ are said to be Fitting equivalent if

$$
\left[\begin{array}{lll}
A & 0 & 0 \\
0 & I & 0
\end{array}\right] \sim\left[\begin{array}{lll}
B & 0 & 0 \\
0 & I & 0
\end{array}\right]
$$

for suitable identity matrices $I$ and zero matrices. Hans Fitting [2, Satz 2(a), p. 579] proved that $A$ and $B$ name isomorphic modules if and only if $A$ and $B$ are Fitting equivalent. Since every finitely presented module over $R$ is a direct sum of cyclic modules if and only if every matrix over $R$ is Fitting equivalent to a diagonal matrix, we obtain the following corollary.

(3.9) Corollary. Every matrix over a ring $R$ is Fitting equivalent to a diagonal matrix if and only if every matrix over $R$ is equivalent to a diagonal matrix.

Note that there is no need to mention canonical form here, for if every matrix over $R$ is equivalent to a diagonal matrix, then $R$ is Hermite, whence Bezout, so by Theorem 3.1 diagonal matrices admit diagonal reduction.

4. Adequate rings. Adequate domains were introduced by Helmer in [6]. It had been known that principal ideal domains were elementary divisor rings and Helmer showed that the less restrictive hypothesis that an integral domain be adequate is sufficient. Kaplansky [11, p. 473] began the consideration of adequate rings with zero divisors by showing that an adequate ring whose zero divisors are in the Jacobson radical is an elementary divisor ring. As a result of our discussion of this hypothesis in $\$ 2$ and a theorem of Henriksen (Theorem 4.1(b) stated below), 
we note the somewhat surprising fact that the only adequate rings whose zero divisors are in the Jacobson radical are either integral domains or valuation rings. The following theorem provides two important facts about adequate rings. We record it for easy reference.

(4.1) Theorem. (a) [6, Theorem 8] An adequate ring is an elementary divisor ring if and only if it is an Hermite ring.

(b) [9, Theorem 4] Every nonzero prime ideal of an adequate ring is contained in a unique maximal ideal.

The (b) part provides a complete picture of the spectrum of an adequate ring when considered as a partially ordered set. Since an adequate ring is Bezout, the set of prime ideals contained in a given prime ideal is linearly ordered. Thus if $R$ is an adequate ring and not an integral domain, then the spectrum of $R$ is a disjoint union of totally ordered sets. If $R$ is an adequate integral domain, then the spectrum of $R$ is a union of totally ordered chains with one and only one common element (the zero prime).

For semilocal rings we can completely describe the adequate rings.

(4.2) Theorem. Let $R$ be a semilocal ring. $R$ is adequate if and only if either

(i) $R$ is a finite intersection of pairwise independent valuation domains (with a common quotient field), or

(ii) $R$ is a finite direct sum of valuation rings.

Proof. If $R$ is adequate, it is, of course, Bezout. Let us assume that $R$ is an integral domain. Then $R$ is an intersection of valuation domains $R=\bigcap_{i=1}^{n} R_{M_{i}}$ where $M_{1}, \ldots, M_{n}$ are the maximal ideals of $R$. If $V$ is a valuation overring of $R$ containing both $R_{M_{i}}$ and $R_{M_{j}}$ for some $i \neq j$, then $V=R_{P}$ for a prime ideal $P$ of $R$ which must be contained in both $M_{i}$ and $M_{j}$. Thus $P$ must be the zero prime ideal of $R$ and $V$ is the quotient field of $R$. Thus the valuation domains $R_{M_{i}}$ are pairwise independent. On the other hand if $R$ is not a domain it still has only a finite number of minimal prime ideals and can (by Bezoutness and Theorem 2.2) be written as a direct sum of rings $R_{1}, \cdots, R_{n}$ each of which has a unique minimal prime ideal. Obviously each $R_{i}$ is Bezout, and since each minimal prime ideal of $R$ is contained in a unique maximal ideal, each $R_{i}$ is local. Therefore each $R_{i}$ is a valuation ring.

Now if $R$ is an intersection of a finite number of independent valuation domains, $R$ is a semilocal Prüfer domain [17, Theorem 11.11, p. 38], hence by [9, Theorem 5, p. 119] a Bezout domain. Let $a, b \in R, a \neq 0$. Let $M_{1}, \ldots, M_{j}, M_{j+1}, \cdots, M_{n}$ be the maximal ideals of $R$ labeled so that $b \notin M_{i}$ for $i=1, \ldots, j$ and $b \in M_{i}$ for $i=j+1, \cdots, n$. Let $v_{1}, \cdots, v_{n}$ be the valuations corresponding to the valuation domains $R_{M_{1}}, \cdots, R_{M_{n}}$. By the approximation theorem [20, Theorem 18, p. 47] 
there exist $r, s \in R$ such that

$$
v_{i}(r)= \begin{cases}v_{i}(a), & \text { if } 1 \leq i \leq j, \\ 0, & \text { if } j+1 \leq i \leq n,\end{cases}
$$

and

$$
v_{i}(s)= \begin{cases}0, & \text { if } 1 \leq i \leq j, \\ v_{i}(a), & \text { if } j+1 \leq i \leq n .\end{cases}
$$

Clearly $v_{i}(a)=v_{i}(r s)$ for $i=1, \cdots, n$, so there is a unit $u$ in $R$ such that $a=$ urs. We may assume $a=r s$ without loss of generality. There is no maximal ideal of $R$ containing both $r$ and $b$, hence $(r, b)=(1)$. If $s^{\prime}$ is a nonunit which divides $s$, then $Z\left(s^{\prime}\right) \subseteq Z(s) \subseteq Z(b)$. Thus $\left(s^{\prime}, b\right) \neq(1)$. This proves that $R$ is adequate.

Finally let $R$ be the direct sum of valuation rings $R_{i}$. It is easy to see that $R$ is a Bezout ring. Let $a=\left(a_{1}, \cdots, a_{n}\right)$ and $b=\left(b_{1}, \cdots, b_{n}\right)$ be elements of $R$ with $a \neq 0$. Define elements $r, s \in R$ coordinatewise as follows:

$$
r_{i}=\left\{\begin{array}{l}
1, \text { if } b_{i} \text { is a nonunit in } R_{i}, \\
a_{i}, \text { if } b_{i} \text { is a unit in } R_{i},
\end{array} \quad s_{i}= \begin{cases}a_{i}, & \text { if } b_{i} \text { is a nonunit in } R_{i}, \\
1, & \text { if } b_{i} \text { is a unit in } R_{i}\end{cases}\right.
$$

Clearly $a=r s$ and $(r, b)=(1)$. Let $s^{\prime}=\left(s_{1}^{\prime}, \ldots, s_{n}^{\prime}\right)$ be a nonunit of $R$ which divides $s$; if $s_{i}^{\prime}$ is a nonunit, then $s_{i}$ is a nonunit and hence $b_{i}$ is a nonunit. Thus $\left(s_{i}^{\prime}, b_{i}^{\prime}\right) \neq(1)$ in $R_{i}$. Therefore $\left(s^{\prime}, b\right) \neq(1)$ and we can conclude that $R$ is adequate.

If we replace the semilocal hypothesis in the previous theorem by a finite character condition, we obtain the following partial converse to Theorem 4.1(b).

(4.3) Theorem. Let $R$ be a ring sucb that $Z(a)$ is finite for all $a \notin J(R)$. Then $R$ is an adequate ring if (and only if) $R$ is a Bezout ring and every nonzero prime ideal of $R$ is contained in a unique maximal ideal of $R$.

Proof. If $R$ is semilocal the previous theorem applies. If $R$ has an infinite number of maximal ideals we note (as did Henriksen [7, p. 161]) that $J(R)$ must be a prime ideal. Thus since $J(R)$ is contained in every maximal ideal, $J(R)=0$ and $R$ is an integral domain. Let $a, b \in R, a \neq 0$, and label the maximal ideals $M_{1}$, $\cdots, M_{n}$ that contain $a$ such that $b \notin M_{i}$ for $i=1, \cdots, j$ and $b \in M_{i}$ for $i=j+1$, $\cdots, n$. Let $S=R \backslash \bigcup_{i=1}^{n} M_{i}$. Then $R_{S}$ is a semilocal Bezout domain in which every nonzero prime ideal is contained in a unique maximal ideal; hence $R_{S}$ is adequate. As in the previous theorem, there are $u, v \in R_{S}$ such that in $R_{S}, a=u v$, $(u, b)=(1)$, and if $v^{\prime}$ is a nonunit of $R_{S}$ which divides $v$, then $\left(v^{\prime}, b\right) \neq(1)$. Write $u=m / t$ and $v=n / t$ with $m, n \in R, t \in S$. Back in $R$, let $r=(a, m)$ and $s=(a, n)$. Then $Z(r)=Z(a) \cap Z(m)=\left\{M_{1}, \ldots, M_{j}\right\}$ and $Z(s)=Z(a) \cap Z(n)=\left\{M_{j+1}, \ldots, M_{n}\right\}$. Therefore $(r, b)=(1)$ and if $s^{\prime}$ is a nonunit which divides $s$, then $\left(s^{\prime}, b\right) \neq(1)$ since $Z\left(s^{\prime}\right) \subseteq Z(s) \subseteq Z(b)$. We conclude the proof by showing $(a)=(r s)$, for then 
$a=r s$ (up to a unit multiple). We accomplish this by showing that these ideals are equal locally. Let $M$ be a maximal ideal of $R$. If $a \notin M$, then $r, s \notin M$ and $a, r, s$ are all units in $R_{M}$. If $a \in M$, use the fact that in $R_{S}$ (and thus in $R_{M}$ ) $(r)=(a, m)=(m)$ and $(s)=(a, n)=(n)$ to obtain the desired local equality.

We pause to discuss the behavior of the rings we have been investigating. Bezout rings, Hermite rings, and elementary divisor rings are closed under homomorphic images. If $R$ is the direct product of $R_{i}$ 's for $i$ in any index set, then $R$ is a Bezout ring (respectively, Hermite ring, elementary divisor ring) if and only if each $R_{i}$ is a Bezout ring (respectively, Hermite ring, elementary divisor ring). The behavior of adequate rings is not nearly as nice. Indeed, from Theorem 4.1(b) one can see that if $R$ is an adequate domain with at least two maximal ideals, then $R \times S$ is not adequate for any nonzero ring $S$. We can however establish the following two positive results.

(4.4) Proposition. If $R$ is an adequate ring and $A$ is an ideal of $R$ contained in $J(R)$, then $R / A$ is an adequate ring.

Proof. Let $\bar{a}, \bar{b}$ be elements of $R / A$ with $\bar{a} \neq 0$ which are the images of $a, b \in R, a \neq 0$, under the canonical map. From the fact that $R$ is adequate we get $r, s \in R$ such that $\bar{a}=\bar{r} \bar{s}$ and $(\bar{r}, \bar{b})=1$. Let $\bar{t}$ be a nonunit in $R / A$ which divides $\bar{s}$. Then for some $k \in J(R)$ we have $t$ divides $s+k$. Hence $Z(t) \subseteq$ $Z(s+k)=Z(s)$. Thus $(t, s) \neq(1)$; thus there is a nonunit $u$ in $R$ which divides both $t$ and $s$. Thus $(u, b) \neq(1)$, and hence $(\bar{u}, \bar{b}) \neq(1)$. Since $\bar{u}$ divides $\bar{t}$ we have $(\bar{t}, \bar{b}) \neq(1)$.

(4.5) Proposition. Let $R$ be the direct product of $R_{i}$ 's where $i \in I$ (an index set with more than one element). Then $R$ is an adequate ring if and only if each $R_{i}$ is adequate and for each $R_{i}$ and each $b_{i} \in R_{i}$, there exists $r_{i}, s_{i} \in R_{i}$ such that $r_{i} s_{i}=0,\left(r_{i}, b_{i}\right)=(1)$ and if $s_{i}^{\prime}$ is a nonunit of $R_{i}$ which divides $s_{i}$, then $\left(s_{i}^{\prime}, b_{i}^{\prime}\right) \neq(1)$.

Note that the condition in the sufficiency part is merely the adequate property in each $R_{i}$ for $a=0$.

Proof. Assume that $R$ is an adequate ring. Fix one index $j$ and pick $a_{j} \in R_{j}$ (here $a_{j}$ could be zero). Let $a=\left(a_{i}\right)$ be an element of $R$ defined by $a_{i}=a_{j}$ if $i=j$ and $a_{i}=1$ if $i \neq j$. Let $b_{j} \in R_{j}$ and define $b=\left(b_{i}\right)$ in $R$ similarly. Then $a \neq 0$ and there exist $r=\left(r_{i}\right), s=\left(s_{i}\right)$ in $R$ satisfying the adequate conditions. Thus $r_{j} s_{j}=a_{j}$ and if $i \neq j, r_{i} s_{i}=1$ so $r_{i}, s_{i}$ are units in $R_{i}$. Since $(r, b)=(1)$ we have $\left(r_{j}, b_{j}\right)=(1)$ in $R_{j}$. If $s_{j}^{\prime}$ is a nonunit in $R_{j}$ which divides $s_{j}$, let $s^{\prime}=\left(s_{i}^{\prime}\right)$ be defined by $s_{i}^{\prime}=s_{j}^{\prime}$ if $i=j$ and $s_{i}^{\prime}=1$ if $i \neq j$. Then $s^{\prime}$ is a nonunit of $R$ which divides $s$ and hence $\left(s^{\prime}, b\right) \neq(1)$. But for all $i \neq j, s_{i}^{\prime}=b_{i}=1$ so we conclude that $\left(s_{j}^{\prime}, b_{j}\right) \neq(1)$. Thus $R_{j}$ is adequate and the additional condition holds. 
Now suppose each $R_{i}$ is adequate and that the adequate condition holds for $a=0$ in each $R_{i}$. Let $a=\left(a_{i}\right), b=\left(b_{i}\right)$ be elements of $R$. Choose the appropriate $r_{i}^{\prime}$ 's and $s_{i}$ 's in each $R_{i}$ (the hypothesis allows this even if some of the $a_{i}$ 's are zero). If $r=\left(r_{i}\right)$ and $s=\left(s_{i}\right)$, then clearly $a=r s$ and $(r, b)=(1)$. Let $s^{\prime}=\left(s_{i}^{\prime}\right)$ be a nonunit which divides $s$. For each $i$ such that $s_{i}^{\prime}$ is a nonunit in $R_{i}$ we have $\left(s_{i}^{\prime}, b_{i}\right) \neq(1)$. Thus we can see that $\left(s^{\prime}, b\right) \neq(1)$ and that $R$ is adequate.

Observe that if $R$ is an adequate ring and the adequate condition does not hold when $a=0$, then $R$ is indecomposable (into a direct product of rings).

Valuation rings trivially satisfy the adequate condition, even for $a=0$. It is known [4, Theorem 11, p. 365] that (von Neumann) regular rings are adequate. We see below that regular rings satisfy the adequate condition for $a=0$.

(4.6) Proposition. If $R$ is a regular ring and $b \in R$, then there exist $r, s \in R$ sucb that $0=r s,(r, b)=(1)$, and if $s^{\prime}$ is a nonunit which divides $s$, then $\left(s^{\prime}, b\right) \neq(1)$.

Proof. Since $R$ is regular any principal ideal is idempotent generated. Let $e$ be an idempotent such that $e R=b R$. Then $R=e R \oplus(1-e) R$. Pick $r=1-e$ and $s=e$; they will do the job.

We return now to the problem of when a Bezout ring is adequate and pose the following question. If $R$ is a Bezout domain with the property that every nonzero prime ideal is contained in a unique maximal ideal, is $R$ necessarily an adequate ring?

Our method of approach will be in terms of the group of divisibility of a Bezout domain. The group of divisibility of an integral domain $R$ with quotient field $K$ is the ordered group of nonzero elements of $K$ modulo the units of $R$, ordered by letting the image of $R$ in this group be the positive elements. The group will be written additively. For properties of the group of divisibility see [8] and [18]. The group of divisibility of a Bezout domain is a lattice ordered group (every pair of elements $a, b$ has an infimum, written $a \wedge b$ ). In [8, Theorem 3 , p. 78] Jaffard proves that every lattice ordered group is the group of divisibility of some integral domain and $\mathrm{Ohm}$ in $[18$, Theorem $6.6, \mathrm{p} .590]$ pointed out that the integral domains obtained by Jaffard's construction are Bezout domains.

Let $A$ be a lattice ordered group. For notational convenience, let $A^{+}=$ $\{x \in A \mid x \geq 0\}$. Let us say $A$ is adequate if for every $a, b \in A^{+}$, there exist $r, s \in A^{+}$ such that $a=r+s, r \wedge b=0$, and if $0<s^{\prime} \leq s$ for some $s^{\prime} \in A$, then $s^{\prime} \wedge b \neq 0$. It is easy to see that we have simply translated the Bezout ring adequate condition into the language of ordered groups. We obviously have the fact that a Bezout domain is adequate if and only if its group of divisibility is adequate.

Let $A$ be a lattice ordered group and let $b \in A^{+}$. Set $A_{b}^{+}=\left\{a \in A^{+} \mid a \wedge b=0\right\}$ and $A_{b}=\left\{a_{1}-a_{2} \mid a_{1}, a_{2} \in A_{b}^{+}\right\}$. Noting that if $a_{1} \wedge b=0$ and $a_{2} \wedge b=0$, then 
$\left(a_{1}+a_{2}\right) \wedge b=0$, we see that $A_{b}$ is a lattice subgroup of $A$.

(4.7) Theorem. Let $A$ be a lattice ordered group. Then $A$ is adequate if and only if for eacb $b \in A^{+}, A_{b}$ is a summand of $A$.

Proof. Let $A$ be adequate and choose $b \in A^{+}$. Let $B_{b}^{+}=\left\{a \in A^{+} \mid a^{\prime} \wedge b \neq 0\right.$ whenever $\left.0<a^{\prime} \leq a\right\}$. If $a_{1}, a_{2} \in B_{b}^{+}$and $0<s \leq a_{1}+a_{2}$ for some $s \in A$, then either $s \wedge a_{1} \neq 0$ or $s \wedge a_{2} \neq 0$; hence $s \wedge b \neq 0$ and $a_{1}+a_{2} \in B_{b}^{+}$. Thus $B_{b}=\left\{a_{1}-a_{2} \mid a_{1}, a_{2} \in B_{b}^{+}\right\}$is a lattice subgroup of $A$. Clearly $A_{b} \cap B_{b}=\{0\}$. Since $A$ is adequate, if $a \in A^{+}$, then $a=r+s$ for some $r \in A_{b}$ and $s \in B_{b}$. Since any element of a lattice ordered group is the difference of two positive elements we get $A=A_{b} \oplus B_{b}$.

Conversely suppose $A=A_{b} \oplus B$ for some lattice subgroup $B$ of $A$. Let $a \in A^{+}$and write $a=r+s$ for some $r \in A_{b}^{+}, s \in B^{+}$. Then $r \wedge b=0$. Suppose $0<s^{\prime} \leq s$ for some $s^{\prime} \in A$. Then $s^{\prime} \in B$ and hence $s^{\prime} \wedge b \neq 0$. Thus $A$ is adequate.

It is well known that any lattice ordered group can be lattice embedded into a product of totally ordered groups in such a way that infimums are preserved. Both a direct product and a direct sum of totally ordered groups are adequate groups, as is easily seen. Thus we get the following corollary to the previous theorem.

(4.8) Corollary. Let $R$ be a Bezout domain. Then $R$ is adequate if its group of divisibility is order isomorphic to either a direct sum or direct product of totally ordered groups.

One can check that the groups of divisibility of the adequate domains considered in Theorem 4.3 are isomorphic to a direct sum of totally ordered groups. There are, of course, adequate domains whose group of divisibility does not satisfy the condition of Corollary 4.8. One such example is the Bezout domain constructed by Heinzer and $\mathrm{Ohm}$ in [5, Example 2.2, p. 276]. We have been unable to determine whether or not a complete converse for Henriksen's Theorem 4 (Theorem 4.1(b) above) exists for an integral domain.

[Added in proof. J. W. Brewer, P. F. Conrad and P. R. Montgomery have constructed a lattice ordered group which, in light of (4.7) above, answers negatively the question following (4.6). Their article Lattice-ordered groups and a conjecture for adequate domains will appear soon in Proc. Amer. Math. Soc.]

\section{BIBLIOGRAPHY}

1. N. Bourbaki, Eléments de mathématique. Fasc. XXVII. Algèbre commutative. Chap. 1: Modules plats, Actualités Sci. Indust., no. 1290, Hermann, Paris, 1961. MR 36 \#146.

2. Hans Fitting, Über den Zusammenhang zwischen dem Begriff der Gleichartigkeit zweier Ideale und dem $\ddot{A}$ quivalenzbegriff der Elementarteilertheorie, Math. Ann. 112 (1935/36), 572-582. 
3. Leonard Gillman and Melvin Henriksen, Rings of continuous functions in which every finitely generated ideal is principal, Trans. Amer. Math. Soc. 82 (1956), 366-391. MR 18, 9.

4. - Some remarks about elementary divisor rings, Trans. Amer. Math. Soc. 82 (1956), 362-365. MR 18, 9 .

5. William Heinzer and Jack $\mathrm{Ohm}$, Locally Noetherian conmutative rings, Trans. Amer. Math. Soc. 158 (1971), 273-284. MR 43 \#6192.

6. Olaf Helmer, The elementary divisor theorem for certain rings without chain conditions, Bull. Amer. Math. Soc. 49 (1943), 225-236. MR 4, 185.

7. Melvin Henriksen, Some remarks on elementary divisor rings. II, Michigan Math. J. $3(1955 / 56), 159-163$. MR 19, 1155.

8. Paul Jaffard, Les systèmes d'idéaux, Travaux et Recherches Mathématiques, IV, Dunod, Paris, 1960. MR 22 \#5628.

9. Christian U. Jensen, Arithmetical rings, Acta Math. Acad. Sci. Hungar. 17 (1966), 115-123. MR $32 \# 7577$.

10. Irving Kaplansky, Commutative rings, Allyn and Bacon, Boston, Mass., 1970. MR $40 \# 7234$.

11. - Elementary divisors and modules, Trans. Amer. Math. Soc. 66 (1949), 464491. MR 11, 155.

12. Jean-Pierre Lafon, Anneaux locaux commutatifs sur lesquels tout module de type fini est somme directe de modules monogènes, J. Algebra 17 (1971), 575-591. MR 44 \#202.

13. - Sur les anneaux commutatifs d'Hermite et à diviseurs élémentaires, C. R. Acad. Sci. Paris Sér. A-B 273 (1971), A964-A966. MR 44 \#6675.

14. Joachim Lambek, Lectures on rings and modules, Blaisdell, Waltham, Mass., 1966. MR 34 \#5857.

15. Hideyuki Matsumura, Commutative algebra, Benjamin, New York, 1970. MR 42 $\# 1813$.

16. Marion Moore and Arthur Steger, Some results on completability in commutative rings, Pacific J. Math. 37 (1971), 453-460.

17. Masayoshi Nagata, Local rings, Interscience Tracts in Pure and Appl. Math., no. 13, Interscience, New York, 1962. MR 27 \#5790.

18. Jack Ohm, Semi-valuations and groups of divisibility, Canad. J. Math. 21 (1969), 576-591. MR 39 \#4146.

19. R. B. Warfield, Jr., Decomposibility of finitely presented modules, Proc. Amer. Math. Soc. 25 (1970), 167-172. MR 40 \#243.

20. Oscar Zariski and Pierre Samuel, Commutative algebra. Vol. II, University Series in Higher Math., Van Nostrand, Princeton, N. J., 1960. MR $22 \# 11006$.

DEPARTMENT OF MATHEMATICS, UNIVERSITY OF NEBRASKA, LINCOLN, NEBRASKA 68508 